\title{
An investigation of cold-wire spatial resolution using a DNS database
}

\author{
Y. Xia, W. Abu Rowin, T. Jelly, D. Chung, I. Marusic and N. Hutchins
}

Department of Mechanical Engineering, The University of Melbourne, Victoria 3010, Australia

\begin{abstract}
The effect of spatial resolution of cold-wire anemometry on both the variance and energy spectrum of temperature fluctuations is analyzed through the use of a numerical database. Temperature fluctuation snapshots from a direct numerical simulation (DNS) of a heated smooth-wall turbulent channel flow are spatially averaged in the spanwise direction to simulate the wire filtering. The results show that the wire length does not affect the mean temperature while it significantly attenuates the variance of temperature fluctuations, particularly in the vicinity of the wall. As the filter length grows, the peaks of the one- and two-dimensional energy spectrograms are further attenuated. Limited attenuation is seen when the filter length is smaller than 30 wall units in the vicinity of the wall, whereas, a complete suppression of the near-wall energetic peak is observed when the filter length exceeds 100 wall units.
\end{abstract}

\section{Keywords}

Cold-wire; spatial resolution; energy spectra.

\section{Introduction}

There are numerous challenges in experimentally resolving the smallest scales in flows, especially for wall-bounded turbulent flows where there is a concentration of small-scale energy very close to the wall. The need to adequately resolve these features places a stringent requirement on the spatial and temporal resolution of sensors. In a standard laboratory boundary layer, the spatial resolution of hot-wire anemometry (HWA) sensors, for instance, can significantly limit the accuracy of velocity measurements [11]. The finite dimension of hot-wire sensors under resolves the small-scale turbulence as the sensor averages the velocity along the wire length, $l$ (which for boundary layer measurements is often aligned with the spanwise direction).

Scalar measurements using cold-wire anemometry will also experience a filtering effect owing to the finite sensor length. Practically, the attenuation of temperature fluctuations is often more acute for cold-wires, than attenuation of velocity fluctuations with HWA, since cold-wires require a much longer length-to-diameter ratio $(l / d)$ [14], where $d$ is the wire diameter. Paranthoen et al. [14] demonstrated that a sufficient lengthto-diameter ratio (e.g. $l / d>1000$ ) mitigates the end conduction loss error, which manifests primarily as a temporal resolution issue. However, for a fixed wire diameter these two requirements are conflicting. Maximizing spatial resolution requires a wire with a length that is sufficiently small relative to the viscous length scale $\left(v / U_{\tau}\right.$ where $U_{\tau}$ denotes friction velocity and $v$ is kinematic viscosity of fluid), while minimizing temporal attenuation due to end conduction effects requires that the sensor is very long relative to its diameter (actually five times longer than the $l / d>200$ recommended for HWA). In practice this means that either cold-wire sensors must be constructed with unreasonably small wire diameters (with consequent issues of drift and fragility), or the Reynolds number range of experiments will be severely restricted. More likely, compromises will be made in viscous scaled wire length $l^{+}\left(\equiv l U_{\tau} / \mathrm{v}\right)$ and length-todiameter ratio. For these reasons, before embarking on such a measurement, it is of critical importance that the effect of spa- tial and temporal filtering due to sensor length and end conduction effects $(l / d)$ are fully understood in the context of wallbounded turbulent flows. In the absence of this understanding, it is not possible to design probes to optimize this compromise for a given set of experimental conditions.

The filtering effect of sensor resolution on the measurement of passive scalars has been estimated from theoretical analysis [15], experiment [1] and DNS data [3], with correction schemes based on the assumption of homogeneous isotropic turbulence. However, the applicability of these schemes to wall-bounded turbulent flows is questionable. In this work, we investigate the effect of spatial filtering in the spanwise direction on the second-order statistics and energy spectra of temperature fluctuations in a thermal turbulent boundary layer. Since experimental data will often contain combined effects due to spatial resolution, end conduction effects and anemometer performance and settings, we here utilize a DNS database to isolate the effects of spatial resolution, with a later intention to expand the analysis to include end-conduction and temporal resolution effects.

\section{Numerical procedure and analysis}

DNS of forced convection in smooth-walled turbulent channel flow was performed at a nominal friction Reynolds number of $R e_{\tau}=395$. The continuity and incompressible Navier-Stokes equations were solved numerically, along with the passive scalar transport equation,

$$
\frac{\partial \theta}{\partial t}+\frac{\partial u_{i} \theta}{\partial x_{i}}=\alpha \frac{\partial^{2} \theta}{\partial x_{i} \partial x_{i}}+G
$$

where $i=1,2$, and 3 indicate the $x, y$ and $z$ (streamwise, spanwise and wall-normal) directions, $t$ is the time and $\alpha$ is thermal diffusivity of fluid. To simulate forced convection, a body force term was added to the right-hand side of Equation (1), $G=-u d T / d x>0$, where $u$ is the instantaneous streamwise velocity, $T(x)$ is the mean temperature and $\theta$ is the instantaneous temperature that is solved by the code. An isothermal boundary condition was imposed at the channel walls, $\theta=\Theta_{w}=0$. The effect of the temperature on the fluid properties (e.g. density and viscosity) was neglected, and, as a result, buoyancy effects were not considered. For further details on numerical procedure the reader is referred to Kozul et al. [10] and Macdonald et al. [12]. The number of cells of the computational domain are $240 \times 240 \times 192$ in the streamwise, spanwise, and wall-normal directions, corresponding to the channel length $\left(L_{x} / h\right)$, width $\left(L_{y} / h\right)$, and height $\left(L_{z} / h\right)$ of $6.0 \times 6.0 \times 2.0$, where $h$ denotes half-channel height. The viscous-scaled streamwise and spanwise mesh resolution was $\Delta x^{+}=\Delta y^{+} \approx 10$ while the viscousscaled wall-normal mesh resolution, $\Delta z^{+}$varied from $\approx 0.63$ at the wall to a maximum of 19.0 at the channel centre-line.

To simulate the effect of the wire length on the cold-wire resolved instantaneous temperature fluctuations, the DNS data were spatially averaged in the spanwise direction using a boxcar filter with different simulated viscous-scaled wire lengths $(10<$ $\left.l^{+}<148\right)$. The smallest simulated wire-length $(\approx 10)$ was set by the spanwise mesh resolution of the DNS, and is referred to here as the unfiltered case. Further boxcar filters are applied at 
multiples of $\Delta y^{+}$to yield $l^{+} \approx 20,30,59,79,99$ and 148 . The boxcar filter has previously been shown to be a suitable model for spatial resolution effects of single-normal sensors [4]. The Reynolds number for the currently available DNS database is low $\left(R e_{\tau}=395\right)$ meaning that, for highest simulated $l^{+}$values, $l / h$ will become appreciable. Under such conditions, the simulated sensor will attenuate some outer-scaled energy and the resulting attenuation will be Reynolds number dependent. However, for $l / h \lesssim 0.15\left(l^{+} \lesssim 60\right)$ and in the near-wall and buffer regions, we would expect this effect to be minimal [4], and hence these results should be generally applicable to higher $R e$ flows for $0<z^{+}<100$ and $l^{+} \lesssim 60$.

The mean temperature $\Theta$, the temperature variance $\left\langle\theta^{2}\right\rangle$ and the energy spectra of temperature fluctuations are subsequently computed from the filtered data. The pre-multiplied onedimensional energy spectra was calculated from

$$
\phi_{\theta \theta}\left(k_{y}, z\right)=k_{y}\left\langle\hat{\theta}\left(k_{y}, z\right) \hat{\theta}^{*}\left(k_{y}, z\right)\right\rangle,
$$

while the pre-multiplied two-dimensional energy spectra was computed as follows

$$
\Phi_{\theta \theta}\left(k_{x}, k_{y}, z\right)=k_{x} k_{y}\left\langle\hat{\theta}\left(k_{x}, k_{y}, z\right) \hat{\theta}^{*}\left(k_{x}, k_{y}, z\right)\right\rangle,
$$

here $k_{x}$ and $k_{y}$ are the streamwise and spanwise wavenumbers respectively, \langle\rangle denotes temporal average, $\hat{\theta}$ is the Fourier transform of $\theta$ and the superscript ${ }^{*}$ denotes the complex conjugate.

\section{Filtering effects on mean and variance statistics}

The wall scaled mean temperature $\Theta / \Theta_{\tau}$ at different filtering lengths is shown in figure 1 , where $\Theta_{\tau}$, the friction temperature, is defined as $q_{w}^{\prime \prime} / \rho C_{p} U_{\tau}$ (where $q^{\prime \prime}$ denotes heat flux at the wall, $\rho$ is the fluid density and $C_{p}$ is the specific heat capacity). As expected, the wire length has no influence on $\Theta / \Theta_{\tau}$, where all the profiles overlap with the thermal linear law of the wall $\langle\Theta\rangle / \Theta_{\tau}=P r z^{+}$and the logarithmic law $\Theta / \Theta_{\tau}=\left(P r_{t} / \kappa\right) \ln z^{+}+4.18$ [7], regardless of the wire length. Here, $P r=0.71$ is the molecular Prandtl number, $P r_{t} \cong 0.85$ [8] is the turbulent Prandtl number and $\kappa=0.4$ is the von Kármán constant. The wire length, on the other hand, has a significant effect on the variance of temperature fluctuations $\left\langle\theta^{2}\right\rangle$, as seen in Figure 2. A large attenuation of $\left\langle\theta^{2}\right\rangle / \Theta_{\tau}^{2}$ in the near-wall region is evident as the filter length, $l^{+}$, increases from 10 to 148 This attenuation diminishes with distance away from the wall, eventually collapsing back to the unfiltered result, although this point of collapse shifts further from the wall with increasing $l^{+}$. The peak value of $\left\langle\theta^{2}\right\rangle / \Theta_{\tau}^{2}$ is further from the wall for the least filtered case at $z^{+} \approx 18$ as compared to the inner peak location of velocity turbulence intensity which is commonly reported at $z^{+} \approx 15$. This is presumably due to the thicker diffusive sublayer than the viscous sublayer in the case of $\operatorname{Pr}=0.71$. The attenuation percentage of temperature variance at $z^{+} \approx 18$ $\Delta\left\langle\theta^{2}\right\rangle=\left(\left\langle\theta^{2}\right\rangle_{r}-\left\langle\theta^{2}\right\rangle_{l}\right) /\left\langle\theta^{2}\right\rangle_{r}$, increases gradually with $l^{+}$as shown in the inset of Figure 2, where $\left\langle\theta^{2}\right\rangle_{r}$ is the temperature variance of the unfiltered (most resolved) case at $l^{+} \approx 10$ and $\left\langle\theta^{2}\right\rangle_{l}$ is the corresponding variance with a filter length $l^{+}$. The inset of Figure 2 also shows the attenuation percentage of the velocity variance at $z^{+} \approx 15, \Delta\left\langle u^{2}\right\rangle=\left(\left\langle u^{2}\right\rangle_{r}-\left\langle u^{2}\right\rangle_{l}\right) /\left\langle u^{2}\right\rangle_{r}$, which as expected from the Reynolds analogy exhibits similar behavior to temperature. It is interesting to see in Figure 2 that the cold-wire measurement of $\left\langle\theta^{2}\right\rangle / \Theta_{\tau}^{2}$ of Orlando et al. [13] with $l^{+}=75$ and $l / d=600$ is considerably lower than the current $\left\langle\theta^{2}\right\rangle / \Theta_{\tau}^{2}$ at a comparable $l^{+}=79$, even though their measurement is at larger $R e_{\tau}=800$. This demonstrates that, aside from the spatial filtering, the cold-wire results of Orlando et al. [13] also suffers from a temporal filter resulting from an $l / d$ that is much smaller than the recommended ratio.
Figure 3 illustrates $\Delta\left\langle\theta^{2}\right\rangle$ as a function of $z^{+}$and filter length $l^{+}$. Near the wall, the attenuation which increases with $l^{+}$, retains almost the same level (horizontal contours) for $z^{+} \lesssim 20$. However, beyond this region the degree of attenuation drops, suggesting that longer wires could be employed, with small attenuation, if the focus of the study were temperature fluctuations in the $\log$ region. As an example, for $z^{+}<20$, if attenuation is to be restricted to $10 \%$, a viscous wire length $l^{+}<20$ is required. In the $\log$ region at $z^{+}=100$, this requirement of $10 \%$ attenuation can be met with a sensor length $l^{+}=40$.

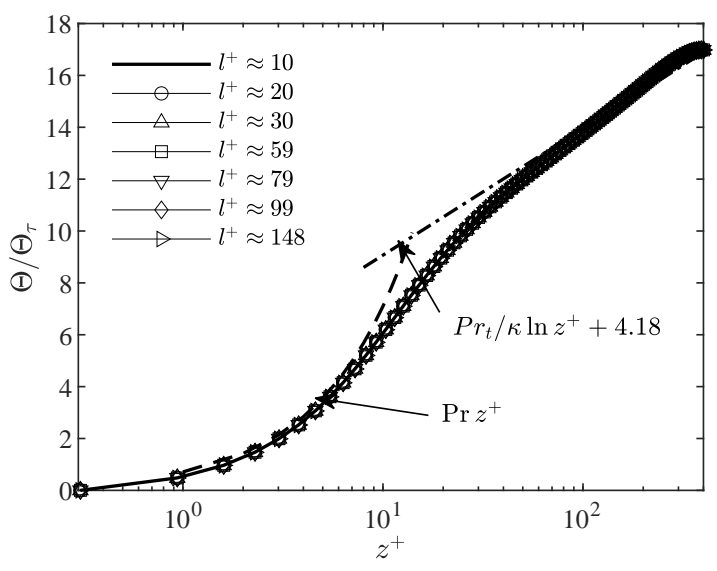

Figure 1. The effect of the filtering length on the mean temperature profile from $10<l^{+}<148$. The thermal linear and logarithmic law of wall are given as dashed and dot-dashed lines respectively.

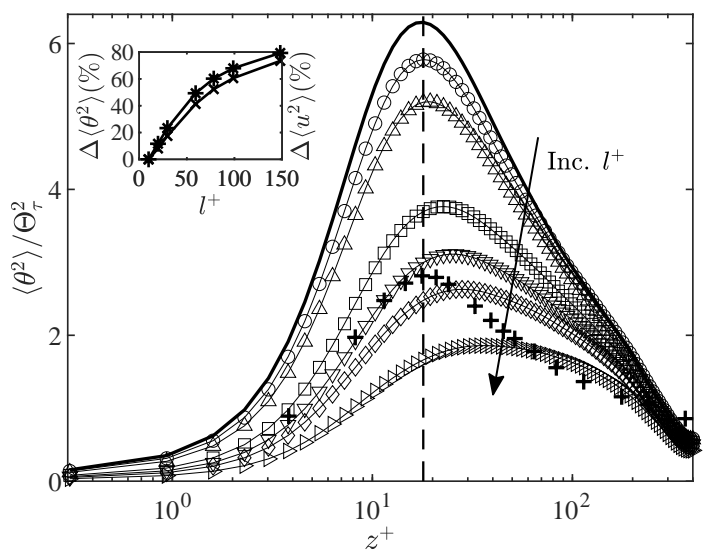

Figure 2. Effect of the filter length on the inner-scaled variance of temperature fluctuations. The dashed line denotes the peak of the unfiltered temperature variance at $z^{+} \approx 18$. Symbols are as defined in Figure 1 . The measured temperature variance data of Orlando et al. [13] with $l^{+}=75$ and $l / d=600$ is shown with + symbols. The inset shows $\Delta\left\langle\theta^{2}\right\rangle(\times)$ at $z^{+} \approx 18$ and $\Delta\left\langle u^{2}\right\rangle(*)$ at $z^{+} \approx 15$ as a function of $l^{+}$.

\section{Filtering effects on energy spectra}

The pre-multiplied one-dimensional energy spectra of the temperature fluctuations, obtained from Equation (2) and scaled with $\Theta_{\tau}^{2}$, is shown in Figure 4 as a function of the spanwise wavelength $\left(\lambda_{y}^{+}\right)$and wall-normal distance $z^{+}$. The horizontal dashed lines are at $\lambda_{y}^{+}=2 l^{+}$for each case, showing the approximate expected influence of the applied boxcar filter. The symbol $\times$ marks the energetic peak of the unfiltered case at $\lambda_{y}^{+} \approx 110$ and wall-normal distance $z^{+} \approx 18$ and it is consistent with the temperature variance peak location in Figure 2 and also comparable to the reported value for the spanwise spacing of the 


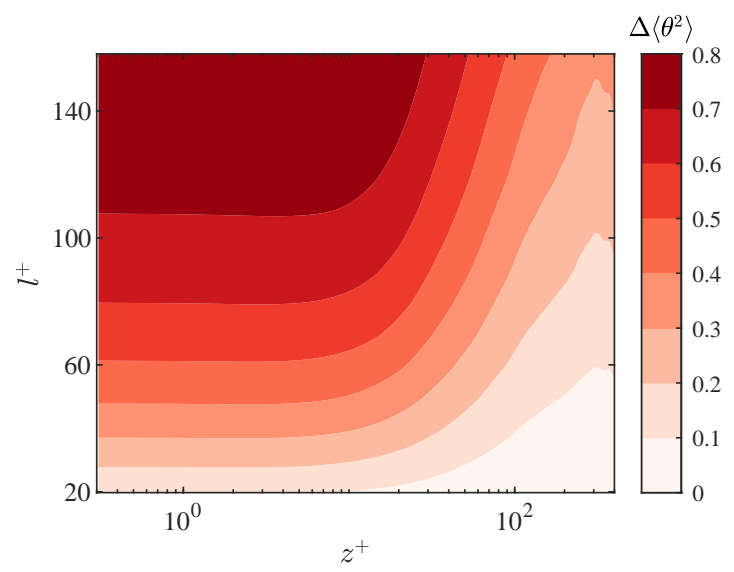

Figure 3. Attenuation percentage $\left(\Delta\left\langle\theta^{2}\right\rangle\right)$ as a function of wall location $z^{+}$and filtered length $l^{+}$.

near-wall streaks $\left(100 \frac{v}{U_{\tau}}[9]\right)$. No significant spatial filtering on the energy spectra is observed when the filter length is $l^{+} \approx 30$ as displayed in Figure 4(b). At higher filter length $\left(l^{+}>59\right)$, the spatial filtering becomes more prominent particularly for the small scales (see Figure 4(c) and (d)). This is evident as the filter length (horizontal dashed line) is shifting upward reaching the peak of the unfiltered case.
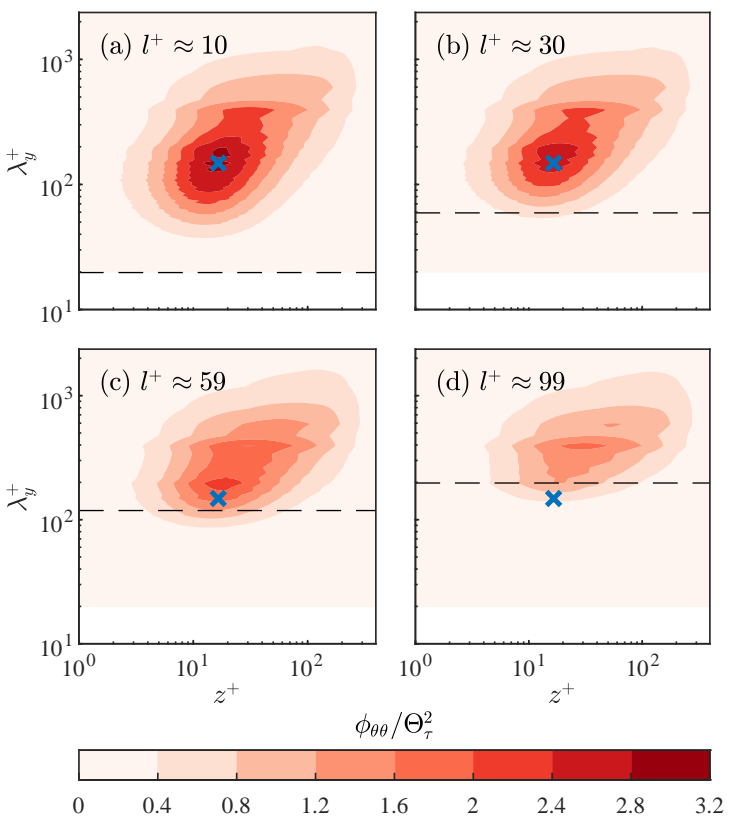

Figure 4. Pre-multiplied one-dimensional energy spectra of the temperature fluctuation $\left(\phi_{\theta \theta} / \Theta_{\tau}^{2}\right)$ as a function of $\lambda_{y}^{+}$against wall distance with different filter length $(a) l^{+} \approx 10$ (unfiltered), $(b) l^{+} \approx 30,(c) l^{+} \approx 59$ and $(d) l^{+} \approx 99$. $\times$ is marked at $\lambda_{y} \approx 110$ at location of $z^{+} \approx 18$. The horizontal dashed line shows $\lambda_{y}^{+}=2 l^{+}$.

The pre-multiplied two-dimensional energy spectra of the temperature fluctuation at $z^{+} \approx 18$, obtained from Equation (3), is shown in Figure 5 as a function of the streamwise $\left(\lambda_{x}^{+}\right)$ and spanwise wavelengths $\left(\lambda_{y}^{+}\right)$. The $\times$symbols in Figure 5 show the energetic peak of the unfiltered case which occurs at $\lambda_{x}^{+} \approx 800$ and $\lambda_{y}^{+} \approx 110$. The wavelengths are comparable to reported values for the near-wall energetic peak of velocity fluctuations at $\lambda_{x}^{+} \approx 1000$ [5] with a spanwise "Kline" scaling of $100 \frac{v}{U_{\tau}}$ [9]. Once the filter length reaches $l^{+} \approx 99$, as seen in Figure $5(d)$, this energetic peak is almost completely attenu-

ated.
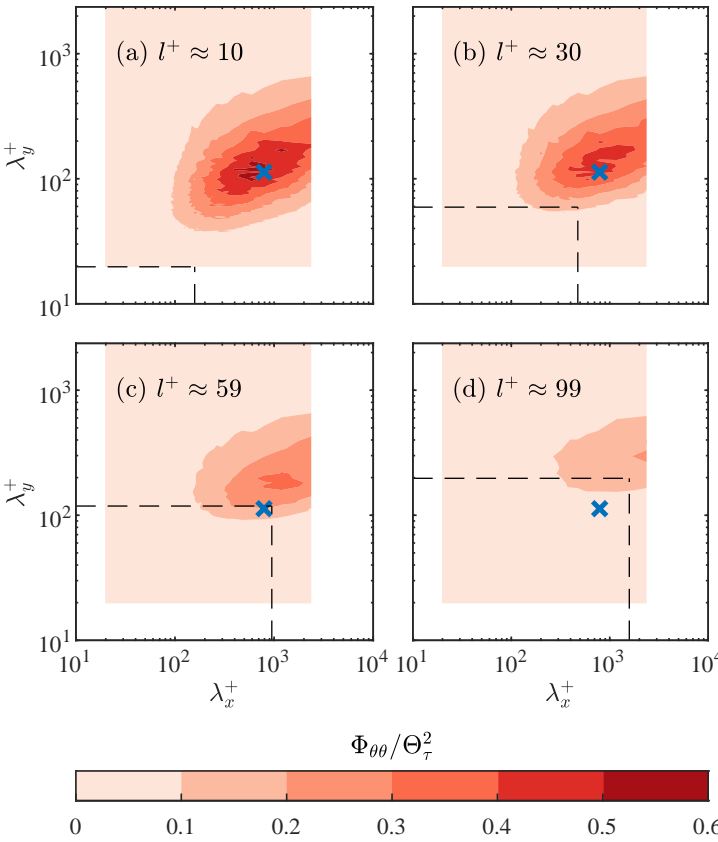

Figure 5. Pre-multiplied two-dimensional energy spectra of temperature $\left(\Phi_{\theta \theta} / \Theta_{\tau}^{2}\right)$ as a function of streamwise wavelength $\left(\lambda_{x}\right)$ and spanwise wavelength $\left(\lambda_{y}^{+}\right)$with filter length $(a) l^{+} \approx 10$ (unfiltered), (b) $l^{+} \approx 30$, (c) $l^{+} \approx 59$ and $(d) l^{+} \approx 99$ at the wall-normal location of $z^{+} \approx 18$. $\times$ is marked at $\lambda_{x}^{+} \approx 800$ and $\lambda_{y}^{+} \approx 110$. The horizontal dashed line shows $\lambda_{y}^{+}=2 l^{+}$and the vertical dashed line shows $\lambda_{x}^{+}=16 l^{+}$.

The effect of the spatial filter can be highlighted by calculating the missing energy in the pre-multiplied spectra, $\chi$, computed by subtracting the filtered $\Phi_{\theta \theta} / \Theta_{\tau}^{2}$ from the unfiltered $\Phi_{\theta \theta} / \Theta_{\tau}^{2}$ [6]. The effects of insufficient spatial resolution on $\chi$ are shown in Figure 6 as a function of $\lambda_{x}^{+}$and $\lambda_{y}^{+}$for $l^{+} \approx 30,59,99$ and 148. The energy peak of the unfiltered case is annotated in Figure $6(\times$ symbols $)$ as defined in Figure 5 . As expected, the relatively small filter length $l^{+} \approx 30$ exhibits little attenuation of energy as shown in Figure 6(a). However, the spatial attenuation becomes evident at larger $l^{+}$, in particular, when the filter length exceeds $l^{+}>99$, in which case the center of the missing energy almost completely overlaps with the energy peak location of the unfiltered case, and the fluctuations are massively under-resolved.

Spatially averaging of DNS grids in the spanwise direction will filter out certain turbulent structures. When considering the 2D energy spectra as shown in Figures 5 and 6, it is important to realize that this spanwise filter will also have an effect in $\lambda_{x}$. Near the wall, turbulent streaky structures, that presumably account for the energetic peak marked by the $\times$ symbol in Figure 5 (and also the peak in broadband variance in Figure 2), have a lengthto-width aspect ratio of approximately $8: 1$. We might also expect the spanwise boxcar filter to strongly affect scales up to twice the filter [16]. These approximate rules of thumb, permit us to demarcate a nominal zone in the 2D spectra, within which we would expect to see strong attenuation of energy. These limits are marked by the dashed lines in Figure 5 and 6. Clearly, in Figure 5, the energy within this region is substantially attenuated, and hence the missing energy shown in Figure 6 is predominantly contained within this region.

Insufficient $l / d$ would be expected to effect the temporal response of the sensor [2], and would further shift this region where we expect substantial attenuation to the right (to higher 
$\lambda_{x}^{+}$). The effect of $l / d$ is the next step required of this research, based on both an analysis of the temporally resolved DNS data with a modeled cold-wire probe and anemometer, and also experimental measurement in a heated smooth wall turbulent boundary layer with different cold-wire sensors of matched $l^{+}$and varying $l / d$.
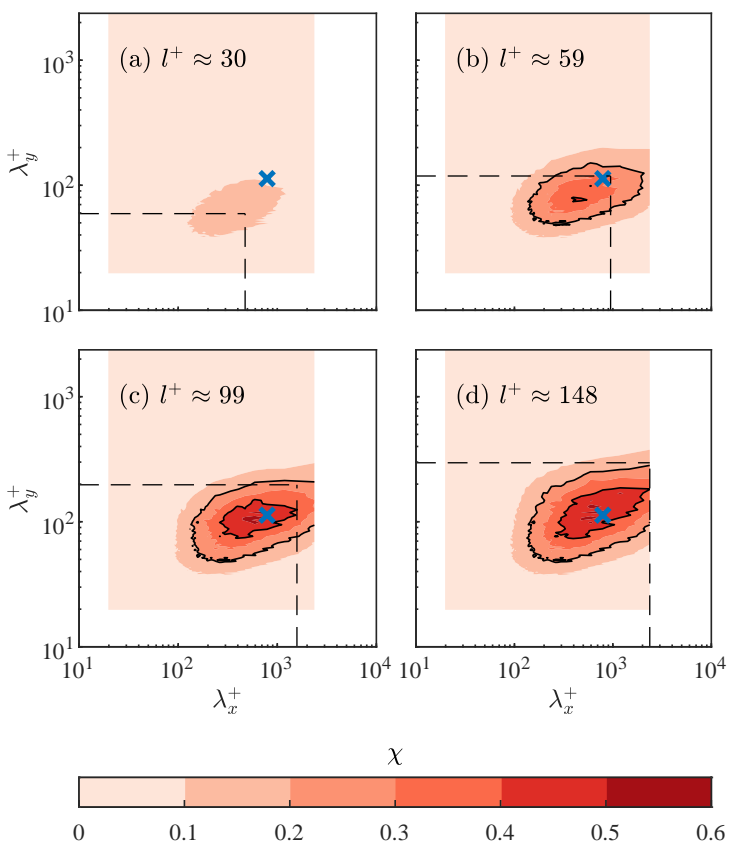

Figure 6. Pre-multiplied two-dimensional missing energy spectra $(\chi)$ of temperature at $z^{+} \approx 18$ for $(a) l^{+} \approx 30(b) l^{+} \approx 59,(c) l^{+} \approx 99$, and $(d)$ $l^{+} \approx 148$. Contours are from $\chi=0.2$ with increments of 0.2 . Horizontal and vertical dashed lines show $\lambda_{y}^{+}=2 l^{+}$and $\lambda_{x}^{+}=16 l^{+}$respectively. The $\times$ symbol is at the energy peak at $\lambda_{x}^{+} \approx 800$ and $\lambda_{y}^{+} \approx 110$.

\section{Conclusions}

A DNS database of forced convective heat transfer within a smooth wall turbulent channel flow has been used to investigate issues of spatial attenuation associated with measurements of temperature fluctuations using cold-wire sensors. Over smooth surfaces and with air as the working fluid $(P r=0.71)$, spatial attenuation of resolved temperature fluctuations due to finite dimension cold-wire sensors behaves very similarly to the attenuation of velocity fluctuations with hot-wire sensors. For rough heated surfaces, where Reynolds analogy is known to break down [12], there will be increasing differences between the attenuation behavior of hot-wires and cold-wires at matched viscous-scaled sensor length. The strictest requirement for small scale sensors occurs near the wall, where $l^{+} \lesssim 20$ is required for errors of less than $10 \%$ in broadband intensity. If our interests lie in the larger scale turbulent fluctuations in the logarithmic region, however, the results here suggest that a probe of length $l^{+} \approx 40$ will have an approximate error in broadband energy of $10 \%$ at $z^{+}=100$. Pre-multiplied two-dimensional energy spectra suggest that a probe of length $l$ will severely filter temperature fluctuations that have a spanwise wavelength $\lambda_{y}<2 l$ and/or a streamwise wavelength $\lambda_{x}<16 l$. The true performance of a cold-wire sensor is a compromise between spatial and temporal resolution. The next step in this research will be to test the effect of end-conduction effects (due to insufficient $l / d$ ) on the two-dimensional energy spectra of temperature fluctuations, with the overall aim of designing optimal cold-wire sensors to minimize attenuation for resolving specified turbulent scales of temperature fluctuations at given wall-normal lo- cations within the thermal turbulent boundary layer.

\section{Acknowledgements}

The authors acknowledge support from the Australian Research Council via grant DP200100969.

\section{References}

[1] Antonia, R. A. and Mi, J., Corrections for velocity and temperature derivatives in turbulent flows, Exp. Fluids, 14, 1993, 203-208.

[2] Arwatz, G., Bahri, C., Smits, A. J. and Hultmark, M., Dynamic calibration and modeling of a cold wire for temperature measurement, Meas. Sci. Technol., 24, 2013, 125301.

[3] Burattini, P., Kinet, M., Carati, D. and Knaepen, B., Corrections for underresolved scalar measurements in turbulent flows using a DNS database, Exp. Fluids, 43, 2007, 31-37.

[4] Chin, C., Hutchins, N., Ooi, A. and Marusic, I., Spatial resolution correction for hot-wire anemometry in wall turbulence, Exp. Fluids, 50, 2011, 1443-1453.

[5] Hutchins, N. and Marusic, I., Evidence of very long meandering features in the logarithmic region of turbulent boundary layers, J. Fluid Mech., 579, 2007, 1-28.

[6] Hutchins, N., Nickels, T. B., Marusic, I. and Chong, M., Hot-wire spatial resolution issues in wall-bounded turbulence, J. Fluid Mech., 635, 2009, 103.

[7] Kader, B., Temperature and concentration profiles in fully turbulent boundary layers, Int. J. Heat Mass Tranf., 24, 1981, 1541-1544.

[8] Kays, W. M., Turbulent Prandtl Number-Where Are We?, J. Heat Transf., 116, 1994, 284-295.

[9] Kline, S. J., Reynolds, W. C., Schraub, F. A. and Runstadler, P. W., The structure of turbulent boundary layers, J. Fluid Mech., 30, 1967, 741-773.

[10] Kozul, M., Chung, D. and Monty, J. P., Direct numerical simulation of the incompressible temporally developing turbulent boundary layer, J. Fluid Mech., 796, 2016, $437-472$.

[11] Ligrani, P. and Bradshaw, P., Spatial resolution and measurement of turbulence in the viscous sublayer using subminiature hot-wire probes, Exp. Fluids, 5, 1987, 407-417.

[12] MacDonald, M., Hutchins, N. and Chung, D., Roughness effects in turbulent forced convection, J. Fluid Mech., 861, $2019,138-162$.

[13] Orlando, A. D., Turbulent transport of heat and momentum in a boundary layer subjected to deceleration, suction and variable wall temperature, Technical Report HMT-17.

[14] Paranthoen, P., Petit, C. and Lecordier, J.-C., The effect of the thermal prong-wire interaction on the response of a cold wire in gaseous flows (air, argon and helium), J. Fluid Mech., 124, 1982, 457-473.

[15] Wyngaard, J., Spatial resolution of a resistance wire temperature sensor, Phys. Fluids, 14, 1971, 2052-2054.

[16] Zurflueh, E. G., Applications of two-dimensional linear wavelength filtering, Geophysics, 32, 1967, 1015-1035. 


\section{University Library}

\section{- M M I N E R VA A gateway to Melbourne's research publications}

Minerva Access is the Institutional Repository of The University of Melbourne

Author/s:

Xia, Y;Rowin, W;Jelly, T;Chung, D;Marusic, I;Hutchins, N

Title:

An investigation of cold-wire spatial resolution using a DNS database

Date:

2020-12-11

Citation:

Xia, Y., Rowin, W., Jelly, T., Chung, D., Marusic, I. \& Hutchins, N. (2020). An investigation of cold-wire spatial resolution using a DNS database. Proceedings of the 22nd Australasian Fluid Mechanics Conference AFMC2020, The University of Queensland. https:// doi.org/10.14264/c2cbd77.

Persistent Link:

http://hdl.handle.net/11343/288706

License:

CC BY-NC 